\title{
AVALIAÇÃO DE SUBSTÂNCIAS NA PRESERVAÇÃO DE NEMATÓIDES ENTOMOPATOGÊNICOS (RHABDITIDA: HETERORHABDITIDAE, STEINERNEMATIDAE) EM DIFERENTES TEMPERATURAS
}

\author{
V. Andaló ${ }^{1}$, R.S. Cavalcanti ${ }^{1}$, J.P. Molina Acevedo ${ }^{2}$, A. Moino Junior ${ }^{1}$ \\ ${ }^{1}$ Universidade Federal de Lavras, Departamento de Entomologia, CP 3037, CEP 37200-000, Lavras, MG, Brasil. \\ E-mail: vanessaandalo@prpg.ufla.br
}

\section{RESUMO}

\begin{abstract}
Foi avaliado o uso de diferentes substâncias com potencial conservante no armazenamento dos nematóides entomopatogênicos Steinernema carpocapsae A11e Heterorhabditissp.JPM4.Osnematóides foram mantidos em água destilada (testemunha), sendo os demais tratamentos compostos por água adicionada de: Tween $80^{\circledR}(0,1 \%)$, etileno glicol $(0,1 \%)$, glicerina $(1 \%)$, glicose $(1 \%), \mathrm{CaCO}_{3}(0,1 \%)$, Triton $^{\circledR}(0,1 \%), \mathrm{KMnO}_{4}(0,01 \%)$ e $\mathrm{NaOCl}(0,1 \%)$, ambos armazenados em diferentes temperaturas. As avaliações foram realizadas aos 30, 60, 90, 120, 150 e 180 dias, por meio de contagens dos juvenis infectantes (JI), sendo determinadas a sua viabilidade e infectividade. Constatou-se que a glicerina agiu como substância conservante na temperatura de $28^{\circ} \mathrm{C}$ para os dois nematóides testados e também a $16^{\circ} \mathrm{C}$ para o nematóide $S$. carpocapsae A11. As demais substâncias usadas, mesmo quando mantiveram os nematóides vivos, não preservaram a infectividade.
\end{abstract}

PALAVRAS-CHAVE: Heterorhabditis, Steinernema, controle biológico, persistência, sobrevivência.

\section{ABSTRACT}

EVALUATION OF POTENTIAL PRESERVING SUBSTANCES FOR THE STORAGE OF ENTOMOPATHOGENIC NEMATODES (RHABDITIDA: STEINERNEMATIDAE, HETERORHABDITIDAE). The use of different substances for the storage of the entomopathogenic nematodes Steinernemacarpocapsae A11 and Heterorhabditis sp. JPM4 was evaluated. The nematodes were kept in distilled water (control) and the other treatments were made with water with the addition of: Tween $80^{\circledast}(0.1 \%)$, ethylene glycol $(0.1 \%)$, glycerin $(1 \%)$, glucose $(1 \%), \mathrm{CaCO}_{3}(0.1 \%)$, Triton $^{\circledR}(0.1 \%), \mathrm{KMnO}_{4}(0.01 \%)$ and $\mathrm{NaOCl}(0.1 \%)$. The evaluations were made at $30,60,90,120,150$ and 180 days, counting the number of infective juveniles (IJ) and determining their survival and infectivity. It was found that glycerin acted as a preserving substance at the temperature of $28^{\circ} \mathrm{C}$ for both nematodes and also in $16^{\circ} \mathrm{C}$ for $\mathrm{S}$. carpocapsae A11. The other substances tested, even when they kept the nematodes alive, did not show this effect in relation to infectivity.

KEY WORDS: Heterorhabditis, Steinernema, biological control, persistence, survival.

\section{INTRODUÇÃO}

Diversasespéciesdenematóidesentomopatogênicos (NEPs) são estudadas para uso em programas de controle biológico de pragas. No entanto, sua sobrevivência tem sido muito discutida, uma vez que em condições delaboratório tem ocorrido alta mortalidade em função do tempo de armazenamento (GLAZER, 2002; НATAB; GAUGLER, 1999).

As estratégias usadas pelos NEPs para sobreviver em condições adversas (dessecação, congelamento, radiação ultravioleta, doenças e predação) são pouco conhecidas, podendo estar relacionadas com o processo de quiescência, migração ou permanência no cadáver dos insetos por períodos extensos, porém existem diferenças entre as condições ideais para cada espécie (Westerman, 1999). Assim, o estudo dos parâmetros queinfluenciam a sobrevivência dos NEPséumaspecto importante para sua liberação no campo, a fim de manter a qualidade do inóculo (BROWN;GAUGLER,1997).

A glicerina é comumente usada como um crioprotetor e remove osmoticamente a água do corpo do nematóide. A cutícula nãoé permeável o suficiente para permitirquea glicerina entre nocorpo, reduzindo

${ }^{2}$ Universidade Estadual do Norte Fluminense, Centro de Ciências e Tecnologias Agropecuárias, LPP, Campos dos Goytacazes, RJ, Brasil. 
os danos na membrana das células. Assim, a glicerina tem sido usada na técnica de criopreservação, na qual os nematóides são submetidos à temperatura de $-130^{\circ}$ C,emnitrogêniolíquido, coma finalidadedeminimizar a formação de cristais intracelulares e intercelulares (GlAZER, 2002; KAYA; STOCK, 1997; LeWIS; SHAPIRO-ILAN, 2002). Além disso, é usada como antidessecante a fim deretardar a evaporação da suspensão com nematóides aplicada na folhagem, para controle de insetos-praga reduzindo a dessecação (BROADBENT; OlthOF, 1995).

O uso de substâncias conservantes pode auxiliar na manutenção da viabilidade desses nematóides, prolongando a sua sobrevivência. Desta forma, esse trabalho teve como objetivo avaliar o uso de diferentes substâncias com potencial conservante para duas espécies de NEPs durante o período de armazenamento em diferentes temperaturas.

\section{MATERIAL E MÉTODOS}

\section{Multiplicação e manutenção dos nematóides entomopatogênicos}

Os nematóides Steinernemacarpocapsae A11(Weiser, 1955) Wouts, Mracek, Gerdin \& Bedding 1982 (isolado na Carolina do Norte, EUA) e Heterorhabditis sp. JPM4 (isolado em Lavras, MG, Brasil) foram cultivados no Laboratório de Patologia de Insetos do Departamento de Entomologia da Universidade Federal de Lavras, Minas Gerais, Brasil. Estes foram mantidos em frascos Erlenmeyer em câmara climática do tipo BOD, a $16 \pm 1{ }^{\circ} \mathrm{C}$, em suspensão aquosa de 500 a $1.000 \mathrm{JI} / \mathrm{mL}$.

Os nematóides foram multiplicados em lagartas de Galleria mellonella (Linnaeus, 1758) (Lepidoptera: Pyralidae), criadas no Laboratório de Patologia de Insetos de acordo com metodologia descrita porDUTKY et al. (1964), utilizando dieta artificial modificada por PARRA (1998).

Após a obtenção das lagartas mortas pelos nematóides, estas foram mantidas em câmara seca (MOLINA;LÓPEZ, 2001) por 5 diase, posteriormente, foram transferidas para armadilhas modificadas de White (WHITE,1927) paraseremcoletadososJIs dosnematóides. A quantificação da suspensão de nematóides foi realizada em placas plásticas para testes serológicos ("ELISA") em microscópio estereoscópico.

Viabilidade e patogenicidade de nematóides entomopatogênicos após armazenamento com substâncias com potencial conservante

Os nematóides S. carpocapsae A11 eHeterorhabditis sp. JPM4 foram mantidos em diferentes substâncias visando melhor conservação em armazenamento. Utilizaram-se os produtos Triton $X-100^{\circledR}(0,1 \%)$, glicerina $(1 \%), \mathrm{KMnO}_{4}(0,01 \%)$, Tween $80^{\circledR}(0,1 \%)$, etileno glicol $(0,1 \%)$, glicose $(1 \%), \mathrm{CaCO}_{3}(0,1 \%)$ e $\mathrm{NaOCl}(0,1 \%)$, os quais foram adicionados às suspensões desses nematóides. No tratamento testemunha foi adicionada apenas água destilada.

As suspensões dos nematóides foram incubadas por 30,60, 90, 120, 150 e 180 dias em água parada nas temperaturas de 8,16 e $28^{\circ} \mathrm{C}$, com 24 h de escotofase, concentração de $3.000 \mathrm{JIs} / \mathrm{mL}$ e 5 repetições por tratamento. Cada parcela foi acondicionada em copo plástico de $110 \mathrm{~mL}$ com tampa para cada repetição, com 40 $\mathrm{mL}$ de suspensão para cada copo. As tampas foram perfuradas, deixando um orifício de, aproximadamente, $2 \mathrm{~cm}$ de diâmetro para possibilitar aeração.

Para avaliação, uma alíquota de $0,1 \mathrm{~mL}$ da suspensão decada repetição foi colocada em placa plástica para testes serológicos ("ELISA") e a contagem foi realizada comauxíliodeummicroscópioestereoscópico. Antesda retirada das alíquotas das suspensões para as avaliações, estas foram agitadas a fim de homogeneizá-las. A porcentagem de viabilidade foi obtida contando-se o número de nematóides vivos e mortos.

No teste de patogenicidade, cinco lagartas de $G$. mellonella foram colocadas em uma placa de Petri $(5 \mathrm{~cm})$ compapelfiltronofundo. Umaalíquota de $0,2 \mathrm{mLdecada}$ suspensão foi retirada e aplicada por placa (uma placa por repetição, em um total de 5 repetições). Estas placas foram colocadas em BOD, com temperatura controlada de $24 \pm 1^{\circ} \mathrm{Ce}$, após 3 dias, foi avaliada a porcentagem de mortalidade das lagartas. Os dados obtidos foram submetidos à análise de variância e ao Teste de Scott-Knott $(\mathrm{P}<0,05)$ para comparação entre as médias para as substâncias conservantes e à análise de regressão para avaliação do efeito do tempo de armazenamento.

\section{RESULTADOS}

\section{Viabilidade de juvenis infectantes}

Ocorreu redução na sobrevivência dos JIs ao longo do tempo para os dois nematóides testados, a $8^{\circ} \mathrm{C}, 16^{\circ} \mathrm{C}$ e $28^{\circ} \mathrm{C}$, em todos os tratamentos, porém a $28^{\circ} \mathrm{C}$ o efeito da ação das substâncias utilizadas foi maior quando comparado com o tratamento testemunha (Figs. 1 e 2).

Para S. carpocapsae A11 ocorreu redução acentuada na sobrevivência a partir dos 60 dias de armazenamento na temperatura de $28^{\circ} \mathrm{C}$. Todavia, no tratamento com glicerina, a sobrevivência dos JIs foi diminuindo gradativamente ao longo do tempo. $\mathrm{O}$ mesmo ocorreu na temperatura de $16^{\circ} \mathrm{C}$, de forma menos acentuada. Para a temperatura de $8^{\circ} \mathrm{C}$, o uso de substâncias para prolongar a sobrevivência dos JI não foi eficaz, com curvas de regressão similares e sobrevivência da testemunha maior do que a dos demais tratamentos (Fig. 1). 


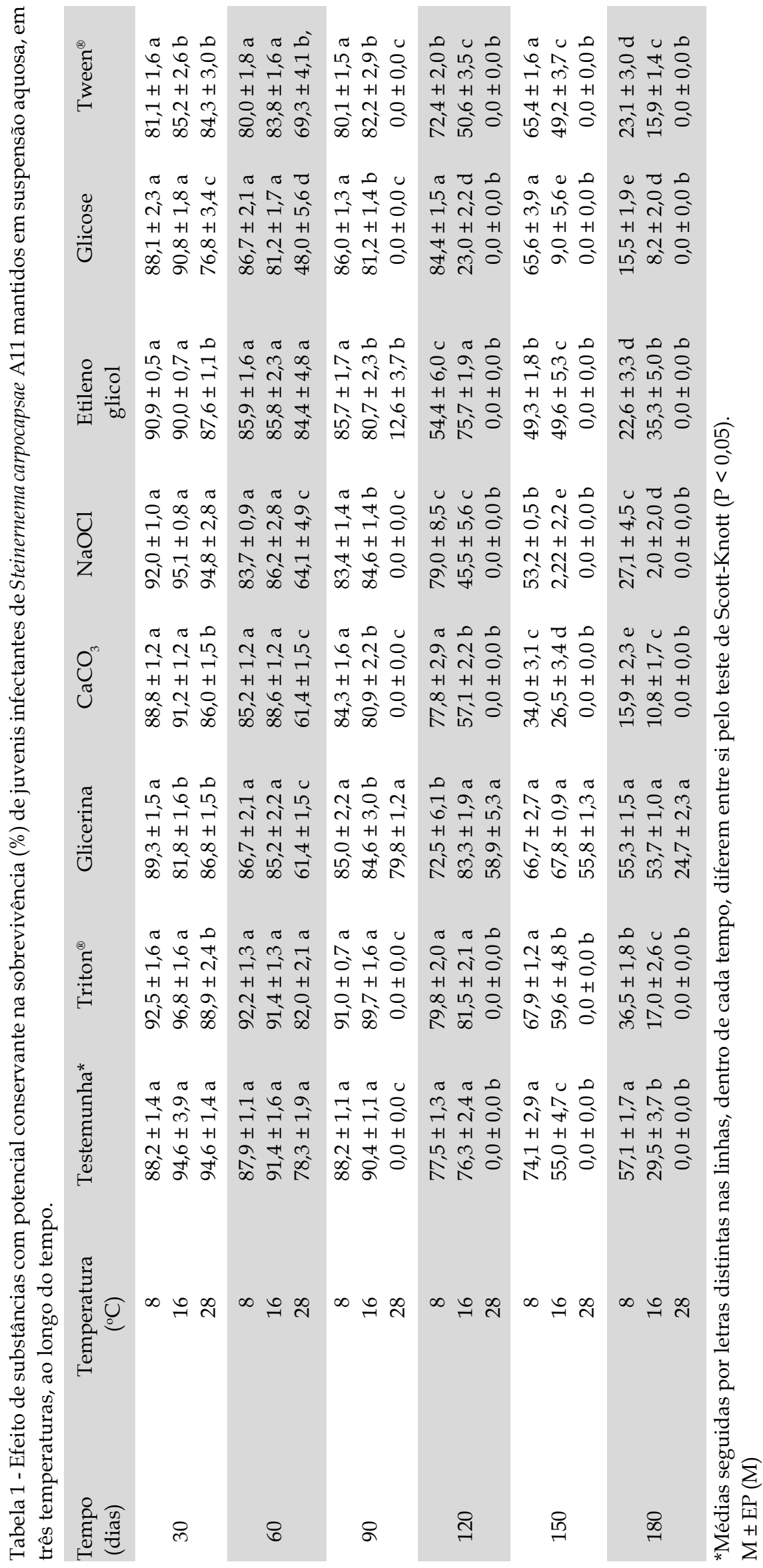


Quando se utilizou $8^{\circ} \mathrm{C}$, houve diferença significativa entre os tratamentos a partir de 120 dias. No entanto, aos 180 dias, apenas a testemunha e o tratamento glicerina proporcionaram sobrevivência maior, sem diferença entre si, evidenciando que não houve melhoria na condição de armazenamento nessa temperatura (Tabela 1). A $16^{\circ} \mathrm{C}$ houve diferença entre os tratamentos desde o princípio das avaliações, eaos 180 dias o tratamento glicerina diferiu dos demais, mantendo maior a sobrevivência dos JIs.

Aos $28^{\circ} \mathrm{C}$ os tratamentos não apresentaram nematóides vivos a partir dos 90 dias, exceto os tratamentos glicerina e etileno glicol; aos 120 dias apenas o tratamento com glicerina apresentava JIs vivos, o que foi observado até a última avaliação. Apesar da porcentagem de sobrevivência final ter sido baixa aos $28^{\circ} \mathrm{C}$, essa diferiu dos demais tratamentos mostrando que a adição de glicerina prolongou o período de sobrevivência desses nematóides para essa temperatura e também na temperatura de $16^{\circ} \mathrm{C}$ (Tabela 1).

Para Heterorhabditis sp. JPM4 houve redução acentuada na sobrevivência aos 60 dias a $8^{\circ} \mathrm{C}$ para todos os tratamentos. $\mathrm{O}$ tratamento em que se empregou $\mathrm{NaOCl}$ causou mortalidade dos JI, sendo que aos 60 dias não havia nematóides vivos nas três temperaturas testadas. Quando a $16^{\circ} \mathrm{C}$, as curvas de regressão apresentaram as mesmas características, exceto para $\mathrm{NaOCl}$, com redução acentuada da sobrevivência. A $28^{\circ} \mathrm{Co}$ tratamento com glicerina teve a sobrevivência reduzida mais lentamente que para os demais tratamentos, pois enquanto aos 120 dias os demais tratamentos quase não tinham mais JI vivos, o tratamento com glicerina ainda apresentava mais de $50 \%$ de sobrevivência (Fig. 2).

Quando a $8^{\circ} \mathrm{C}$, houve diferença entre os tratamentos somente na avaliação com 30 dias. No entanto, a testemunha, Tween ${ }^{\circledR}$ e Triton ${ }^{\circledR}$ foram os que proporcionaram maior sobrevivência, não havendo diferença entre eles, sem melhoria na condição de armazenamento nessa temperatura. A partir de 60 dias, nessa temperatura, não foram encontrados nematóides vivos (Tabela 2).

A $16^{\circ} \mathrm{C}$ houve diferença entre os tratamentos em todas avaliações. Contudo, não houve diferença entre a testemunha e os demais tratamentos aos 180 dias, exceto pelos tratamentos com $\mathrm{NaOCl}$ e glicose, que apresentaram porcentagem de sobrevivência menor. Aos $28^{\circ} \mathrm{C}$ os tratamentos glicerina, seguidos por etileno glicol e Tween ${ }^{\circledR}$, mantiveram índices maiores de sobrevivência, diferindo da testemunha e dos demais tratamentos aos 90 dias de armazenamento (Tabela 2).
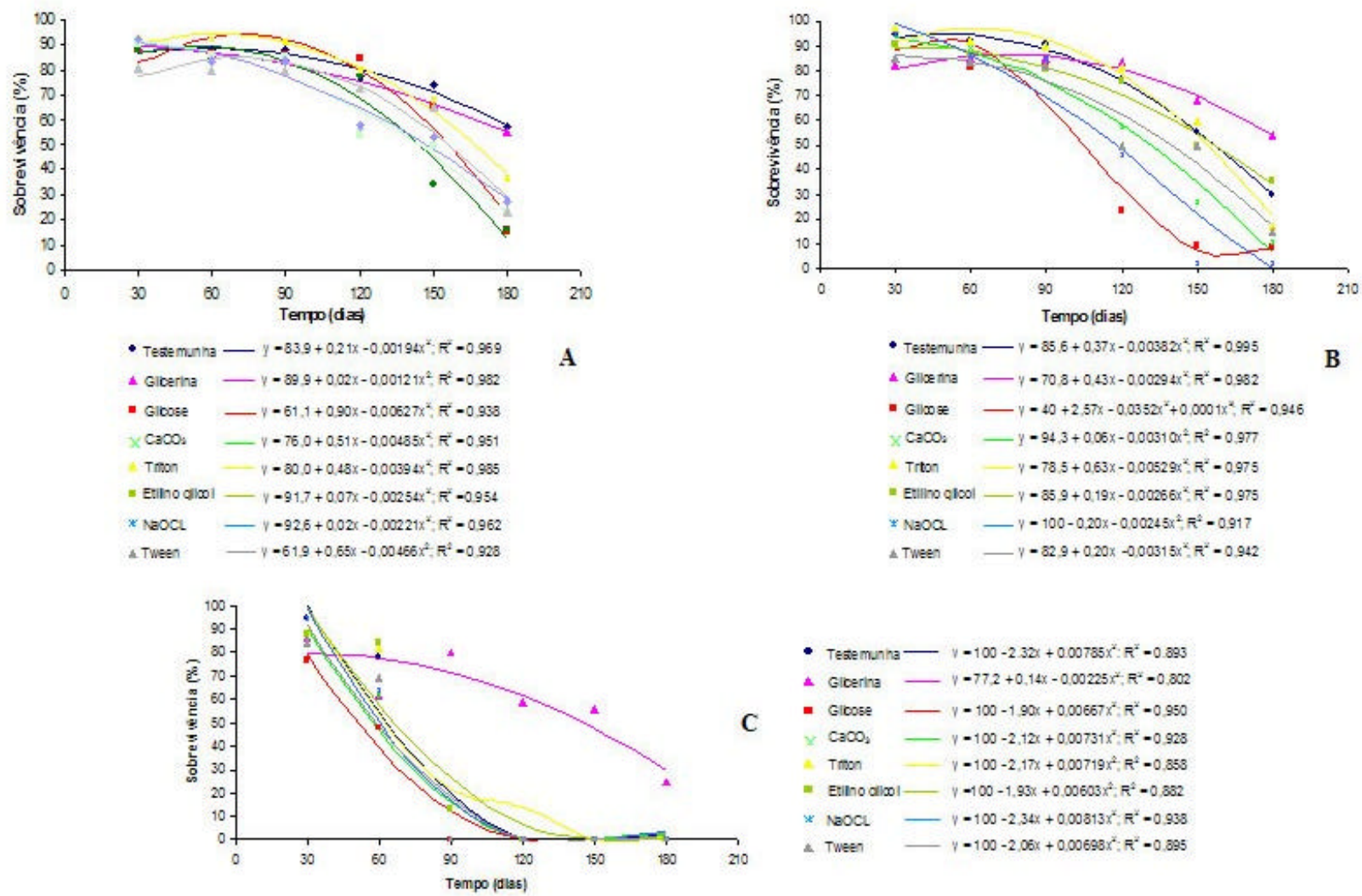

Fig. 1 - Efeito do tempo na sobrevivência (\%) de juvenis infectantes deSteinernemacarpocapsae A11 mantidos em suspensão aquosa com substâncias conservantes, em três temperaturas. A: $8^{\circ} \mathrm{C}, \mathrm{B}: 16^{\circ} \mathrm{C}$ e C: $28^{\circ} \mathrm{C}$. 
Com 120 e 150 dias, apenas o tratamento glicerina manteve os nematóides vivos, e aos 180 dias estes não foram mais encontrados. Apesar dos JIs terem morrido ao final dos 180 dias, observou-se que a sobrevivência foi prolongada quando usada glicerina na temperatura de $28^{\circ} \mathrm{C}$ para Heterorhabditis sp. JPM4 (Tabela 2).

\section{Infectividade de juvenis infectantes}

Houve redução na infectividade dos JIs ao longo do tempo para os dois nematóides nas temperaturas testadas em todos os tratamentos. Porém, quando a $28^{\circ} \mathrm{C}$, o uso de algumas substâncias manteve maior o índice de infectividade do que quando comparado ao tratamento apenas com água. O mesmo não foi observado para as demais temperaturas $\left(8\right.$ e $\left.16^{\circ} \mathrm{C}\right)$, nas quais não foi observado efeito das substâncias testadas comoagentes conservantes nainfectividade(Figs. 3 e 4$)$.

Para S. carpocapsae A11 ocorreu uma redução acentuada na infectividade a partir dos 60 dias de armazenamento na temperatura de $28^{\circ} \mathrm{C}$, com o tratamento glicerina mantendo a infectividade maior do que para os demais tratamentos e a testemunha até o período de armazenamento de 120 dias (Fig. 3C). Esse resultado corresponde ao que foi obtido na curva de regressão do estudo de viabilidade (Fig. 1C), mostrando que a glicerina manteve esses nematóides viáveis e infectantes por um período maior de tempo. O mesmo resultado foi obtido para a temperatura de $16^{\circ} \mathrm{C}$.

Quando em $8^{\circ} \mathrm{C}$, o uso de substâncias para prolongar a infectividade dos JIs não foi eficaz, com curvas similares de regressão entre os tratamentos e a testemunha (Fig. 3A). As curvas de sobrevivência e infectividade foram correspondentes para $16^{\circ} \mathrm{C}$ e $28^{\circ} \mathrm{C}$, mostrando que os JIs mantidos vivos estavam também infectantes, pois pode ocorrer de JIs estarem vivos porém não infectivos, o que não ocorreu nessa situação. Já a $8^{\circ} \mathrm{C}$, a sobrevivência manteve-se por um período longo (57,1\% para a testemunha aos 180 dias), porém a manutenção da infectividade não foi notada (Figs. 1 e 3).

A $8^{\circ}$ Chouve diferença significativa entre os tratamentos. Todavia, aos 180 dias nenhum tratamento sobressaiu-se à testemunha, mostrando que não houve melhoria na condição de armazenamento nessa temperatura (Tabelas 1 e 3 ).
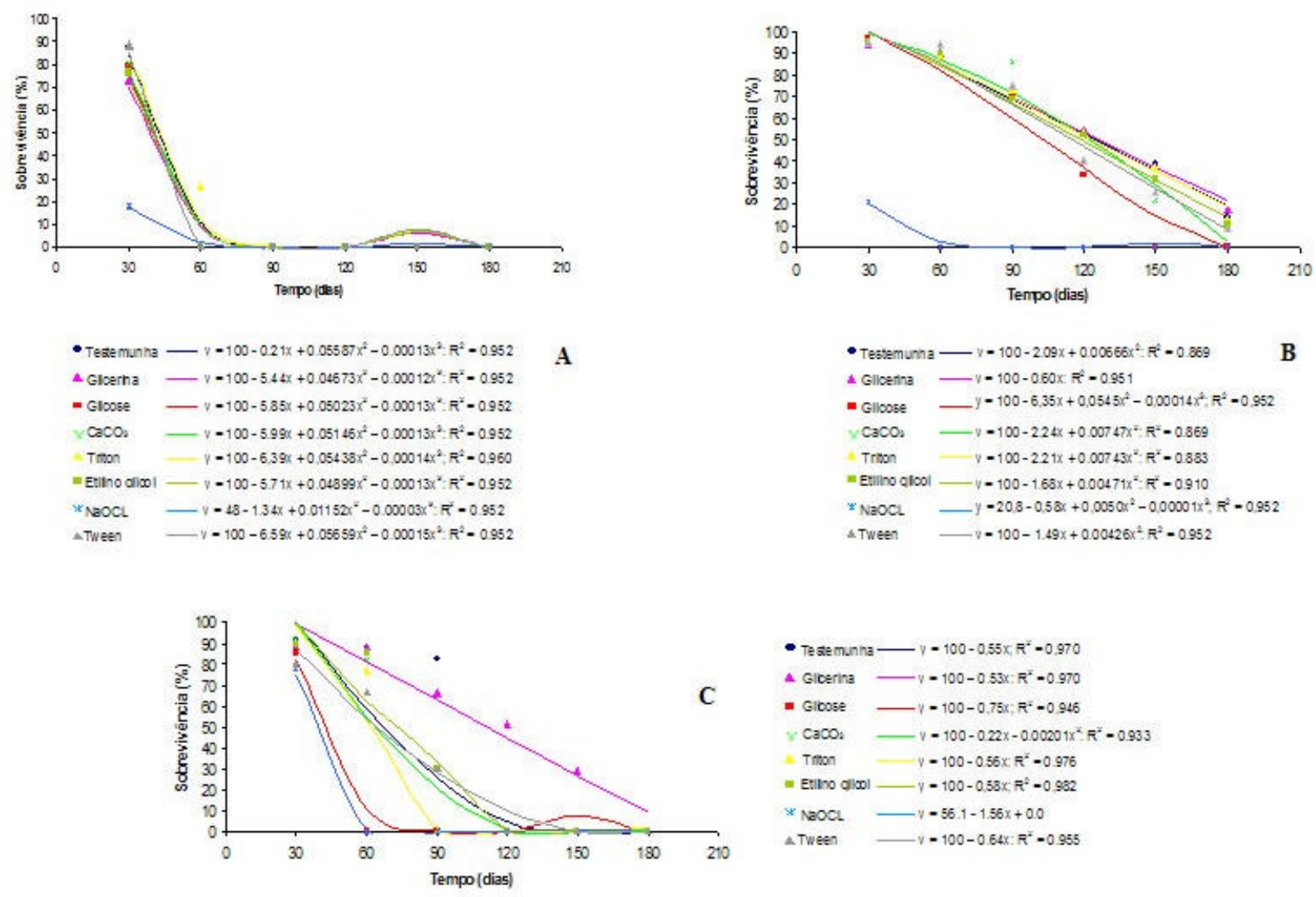

Fig. 2 - Efeito do tempo na sobrevivência (\%) de juvenis infectantes de Heterorhabditis sp. JPM4 mantidos em suspensão aquosa com substâncias conservantes, em três temperaturas. A: $8^{\circ} \mathrm{C}, \mathrm{B}: 16^{\circ} \mathrm{C}$ e C: $28^{\circ} \mathrm{C}$. 


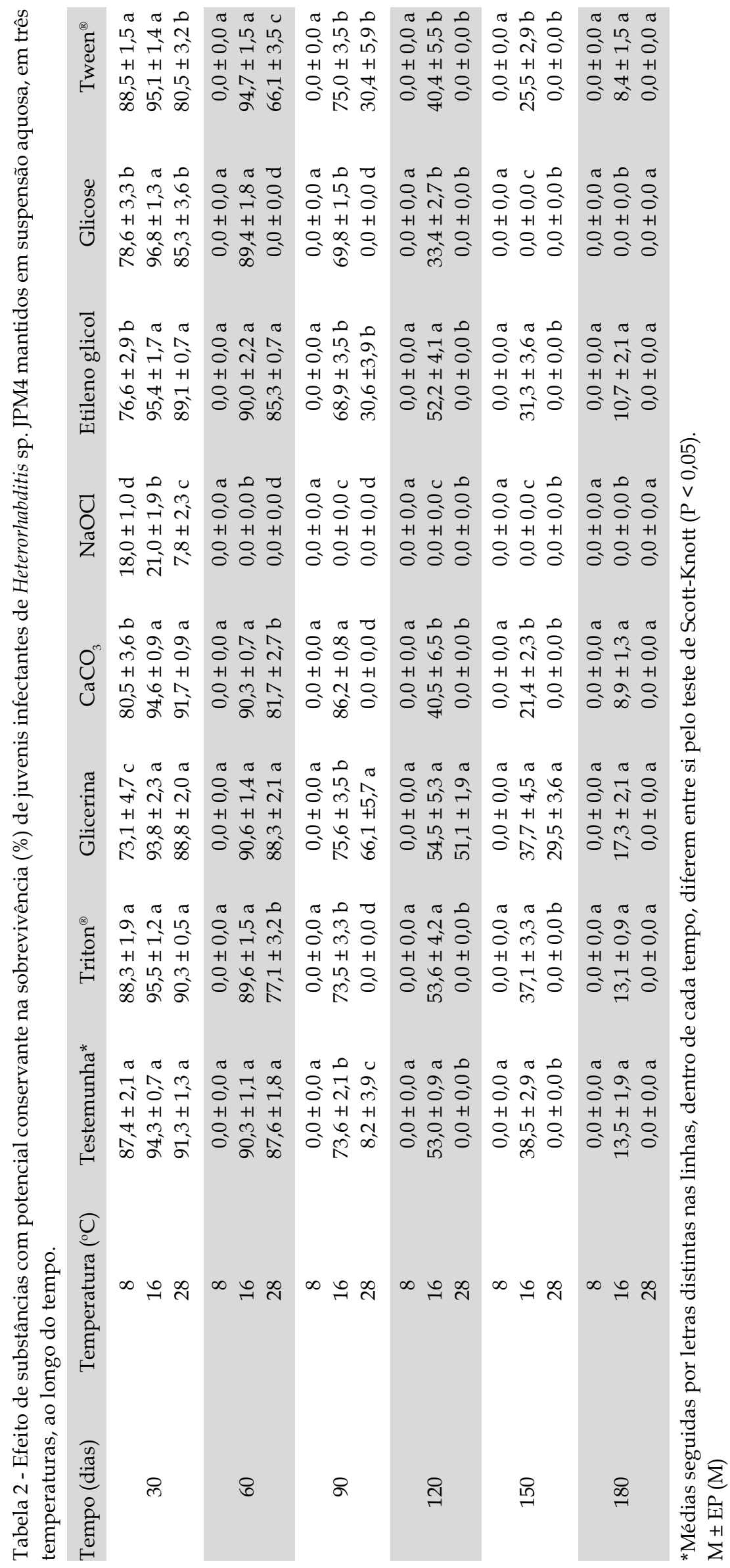



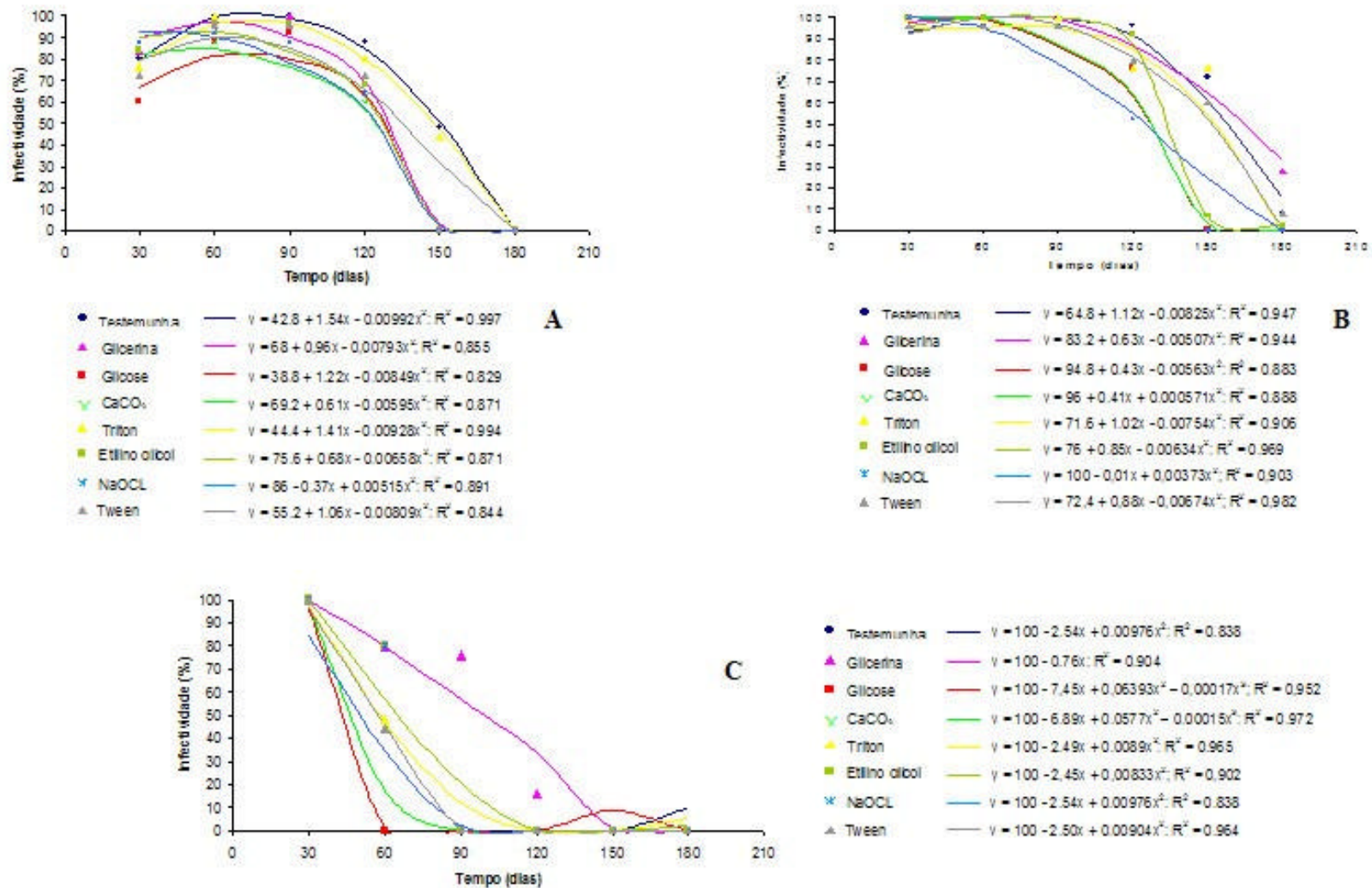

Hig. 3 - Eteito do tempo na intectividade (\%) de juvenis intectantes de Steinernema carpocapsae A11 sobre lagartas deGalleria mellonella mantidos em suspensão aquosa com substâncias conservantes, em três temperaturas. A: $8^{\circ} \mathrm{C}, \mathrm{B}: 16^{\circ} \mathrm{C}$ e C: $28^{\circ} \mathrm{C}$.
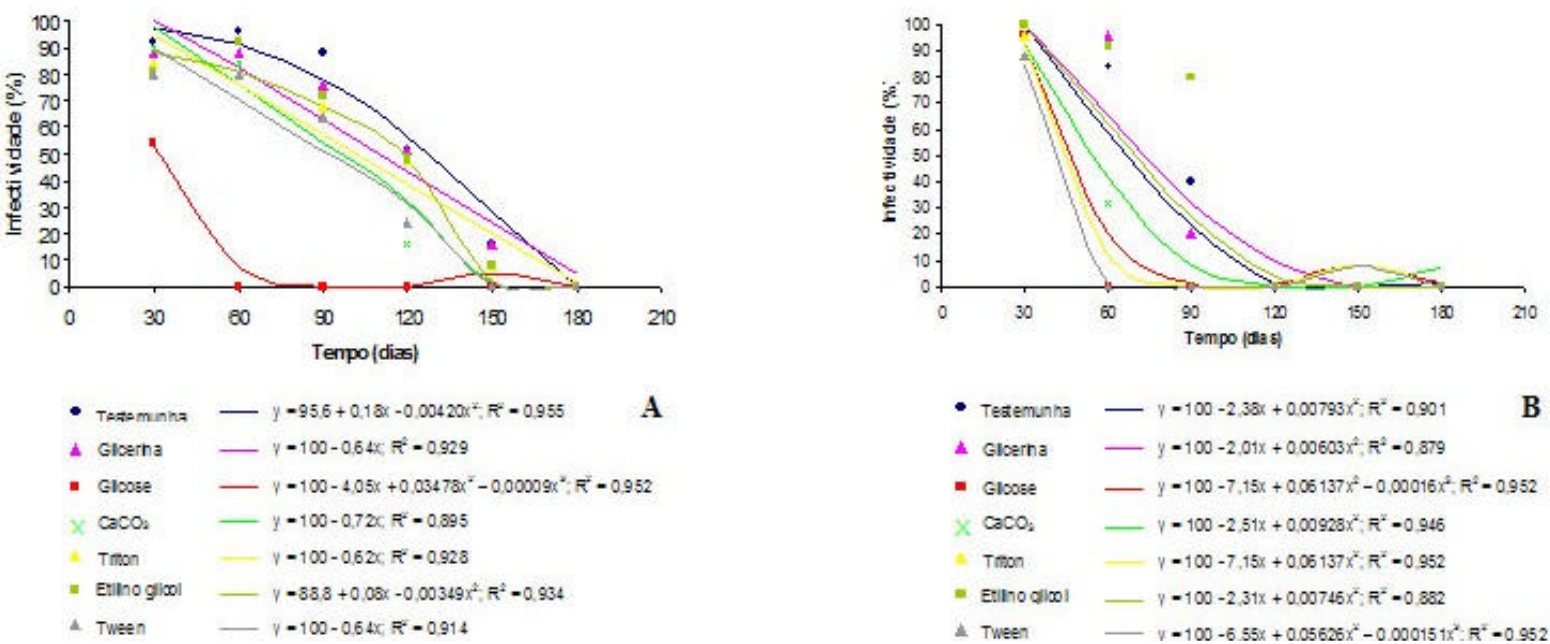

Fig. 4 - Efeito do tempo na infectividade (\%) de juvenis infectantes de Heterorhabditis sp. JPM4 sobre lagartas de Galleria mellonella mantidos em suspensão aquosa com substâncias conservantes, em duas temperaturas. A: $16^{\circ} \mathrm{C}$ e B: $28^{\circ} \mathrm{C}$.

Quando em $16^{\circ} \mathrm{C}$, houve diferença entre os tratamentos a partir de 120 dias, e aos 180 dias os tratamentos glicerina e etileno glicol diferiram dos demais, mantendo maior a infectividade dos JIs. A partir dos 90 dias, na temperatura de $28^{\circ} \mathrm{C}$, os tratamentos não apresentaram nematóides infectantes, exceto o tratamento glicerina, e aos 150 dias não foi encontrado nematóide infectante em nenhum tratamento. Apesar da porcentagem de infectividade ter sido baixa aos $28^{\circ} \mathrm{C}$ no tratamento com glicerina, essa diferiu dos demais, mostrando que a adição de glicerina prolongou o período de infectividade desses nematóides para essa temperatura, sendo esses dados correspondentes aos de sobrevivência (Tabelas 1 e 3). Assim, a adição de glicerina mostrou efeito de preservar a sobrevivência e a infectividade dos JIs a $28^{\circ} \mathrm{C}$, considerada uma temperatura alta para NEPs, diferindo dos demais tratamentos, onde os JIs não se mantiveram infectivos aos 90 dias. 


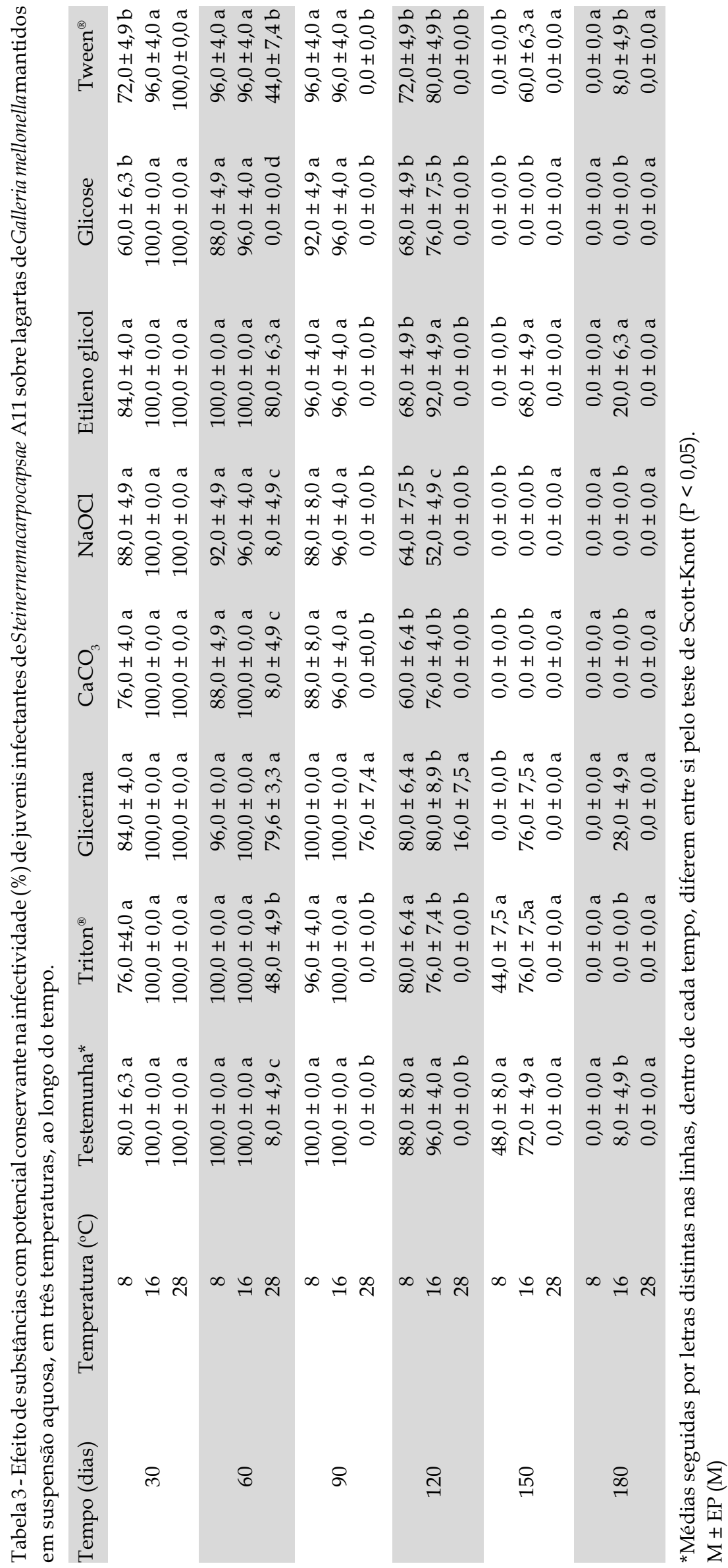




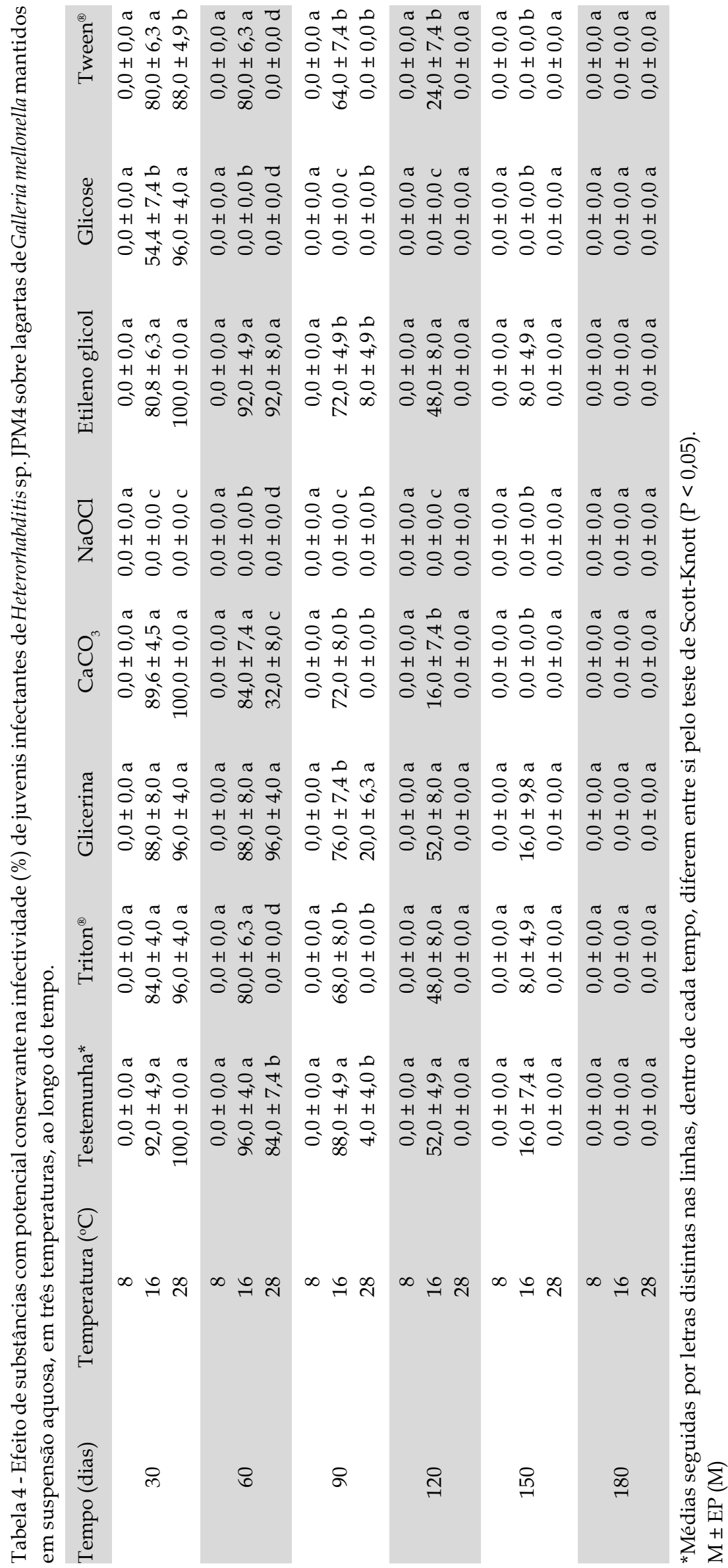


Para Heterorhabditis sp. JPM4 não foi observada infectividade na temperatura de $8^{\circ} \mathrm{C}$ para todos os tratamentos já na primeira avaliação, aos 30 dias, mostrando que mesmo havendo nematóides vivos até os 60 dias de armazenamento (Fig. 2), estes não são mais infectantes (Tabela 4). Isto pode ocorrer pela redução da reserva lipídica e de glicogênio dos JIs, perdendo a capacidade de penetrar no inseto, mesmo estando vivos.

Quando em $16^{\circ} \mathrm{C}$, a redução da infectividade foi gradativa ao longo do tempo, exceto para o tratamento com glicose, com redução acentuada. Além disso, o tratamento com $\mathrm{NaOCl}$ prejudicou a infectividade desde a primeira avaliação aos 30 dias, não causando mortalidade em G. mellonella. Este resultado corresponde ao obtido na avaliação de sobrevivência, uma vez que $\mathrm{NaOCl}$ causou alta mortalidade desses nematóides desde o princípio do armazenamento em todas as temperaturas (Figs. 2 e 4), não sendo adequado para preservar a sobrevivência desse nematóide.

Em $28^{\circ} \mathrm{C}$, os tratamentos reduziram acentuadamente a infectividade até os 90 dias para todos os tratamentos, o que também foi observado para sobrevivência. O tratamento glicerina manteve a sobrevivência elevada até os 150 dias de avaliação, porém, sem manutenção da infectividade (Figs. 2C e 4B). Assim, o uso da glicerina para Heterorhabditis sp. JPM4 foi eficiente até os 90 dias, já que aos 150 dias esses não estavam mais infectantes.

Quando em $8^{\circ} \mathrm{C}$, nenhuma substância testada promoveu melhoria na condição de armazenamento, pois, apesar de terem sido encontrados nematóides vivos, o teste de infectividade não foi positivo. $\operatorname{Em} 16^{\circ}$ $\mathrm{C}$, houve diferença entre os tratamentos a partir dos 90 dias. Entretanto, nenhum tratamento sobressaiuse à testemunha, e aos 150 dias o Triton ${ }^{\circledR}$, glicerina e etileno glicol, apesar de contribuírem para manter a infectividade, não diferiram da testemunha, não havendo efeito dessas substâncias como agentes conservantes para o parâmetro infectividade. Aos $28^{\circ} \mathrm{C}$ houve diferença entre os tratamentos desde a primeira avaliação, e aos 90 dias o tratamento com glicerina diferiu dos demais, mantendo maior a infectividade dos nematóides. O mesmo foi observado aos 120 dias. Porém, aos 150 dias não houve mortalidade em nenhum tratamento testado, apesar da sobrevivência ter sido mantida (Tabelas 2 e 4). Apesar disso, observou-se que a sobrevivência e a infectividade de Heterorhabditis sp. JPM4 foram prolongadas quando usada glicerina na temperatura de $28^{\circ} \mathrm{C}$.

Dessa forma, para o nematóide Heterorhabditissp. JPM4 houve efeito da glicerina quando em $28^{\circ} \mathrm{C}$ para manutenção da sobrevivência e infectividade. $\mathrm{A} 16^{\circ} \mathrm{C}$ não houve efeito dos tratamentos testados, pois glicerina e etileno glicol não diferiram da testemunha quanto à infectividade. Na temperatura de $8^{\circ} \mathrm{C}$ também não ocorreu efeito das substâncias testadas, já que os nematóides morreram no princípio das avaliações e em nenhuma avaliação causaram mortalidade.

Para S. carpocapsae A11 não houve efeito das substâncias testadas na temperatura de $8^{\circ} \mathrm{C}$, pois nenhum tratamento foi melhor que a testemunha. A $16^{\circ} \mathrm{C}$, o tratamento com glicerina prolongou a sobrevivência e infectividade dos nematóides, diferindo da testemunha apenas na última avaliação, aos 180 dias, e com uma baixa porcentagem de infectividade $(28 \%)$. A $28^{\circ} \mathrm{C}$ houve efeito do uso da glicerina, pois esta prolongou a infectividade dos nematóides por 60 dias. No entanto, aos 120 dias a infectividade foi baixa (16\%) e aos 150 dias não havia nematóides vivos (Tabela 3).

Constatou-se que a glicerina agiu como uma substância conservante na temperatura de $28^{\circ} \mathrm{C}$ para os dois nematóides testados e também a $16^{\circ} \mathrm{C}$ para o nematóide S. carpocapsae A11. As demais substâncias usadas, mesmo quando mantiveram os nematóides vivos, não mostraram esse efeito em relação à infectividade, não exercendo uma ação conservante.

\section{DISCUSSÃO}

De acordo com Flanders et al. (1996), diversos são os fatores que influenciam a sobrevivência e infectividade dos nematóides entomopatogênicos, como temperatura, radiação solar, umidade e disponibilidade de oxigênio. Além disso, existem diferenças entre as espécies. Membros da família Heterorhabditidae são mais sensíveis a radiação, produtos químicos, temperatura e dessecação do que os da família Steinernematidae, além de apresentarem pior estabilidade de armazenamento. O mesmo foi observado no presente trabalho, já queS.carpocapsae teve uma redução gradual da sobrevivência e infectividade, enquanto paraHeterorhabditis sp.JPM4 essa diminuição ocorreu rapidamente.

Segundo Glazer (2002), a temperatura extrema é um dos fatores que limita a sobrevivência desses organismos, influenciando tanto na sobrevivência como na mobilidade e infectividade. Assim, Andalóetal. (2005) concluíram que em temperaturas de $8^{\circ} \mathrm{Ce} 12^{\circ} \mathrm{C}$ houve redução de viabilidade e infectividade para os nematóides Heterorhabditis sp. CCA e Heterorhabditis sp. JPM4. Além disso, juvenis infectantes de heterorhabditídios são afetados em baixa temperatura, diminuindo a proporção de nematóides infectantes e a habilidade para procurar hospedeiros (WESTERMAN, 
1999). Dessa forma, esses resultados foram semelhantes aos encontrados nesse trabalho, uma vez que, apesar de terem sido encontrados JIs vivos, muitos já haviam perdido a capacidade de infectar o inseto.

Outro fator que influencia a atividade do nematóide é a mudança na composição química ou microbiana no meio em que ele se encontra (DEMPSEY;GrifFIN, 2002; FItTERs; Griffin, 2004). O uso de substâncias adicionadas na água da suspensão pode ser prejudicial ou favorecer a sobrevivência dos JIs. Nesse trabalho, concluiu-se que a glicerina favoreceu o armazenamento dos JIs na temperatura de $28^{\circ} \mathrm{C}$, no entanto, a $8^{\circ} \mathrm{C}$ a morte dos nematóides pode estar mais associada à adaptação térmica. Na temperatura de $8^{\circ} \mathrm{C}$ a adição de substâncias não teve ação conservante, pois a morte provavelmente está associada à baixa temperatura. GREAL (2000) discute a possibilidade da longevidade desses nematóides a baixas temperaturas ser mais dependente da sua tolerância ao frio do que da quantidade de reservas energéticas. Sendo assim, a glicerina possivelmente agiu dando proteção à cutícula do nematóide a $28^{\circ} \mathrm{C}$. BROWN; GAUGLER (1997) obtiveram sobrevivência aumentada para nematóides congelados em glicerina a $20 \%$ em comparação com o congelamento usando apenas água, confirmando a ação da glicerina como um protetor.

Quando armazenados em água, os NEPs economizam suas reservas de várias formas, mudando seu comportamento locomotor, adotando postura estacionária ou por meio de processo de agregação. Isso possibilita a diminuição do gasto energético e a população supera as condições de estresse (PATEL et al., 1997; Wright et al., 1997; Wright; PERRY, 2002), mantendo-se viva e infectante mesmo em condições adversas a sua sobrevivência.

As fontes de energia dos nematóides são o glicogênio e lipídios, reservas por meio das quais eles irão manter-se vivos até encontrarem um novo hospedeiro para parasitar. A alta taxa de lipídios em JIs permite maior sobrevivência, assim, os nematóides são dependentes de lipídios para um período maior de armazenamento, uma vez que a sobrevivência declina juntamente com a diminuição das reservas dos JIs (HAss et al., 2002; Lewis et al., 1995; MENTi et al., 2003). Ouso da glicerina como conservante teve efeito principalmente em temperatura de $28^{\circ} \mathrm{C}$, quando os nematóides se movimentam bastante e gastam suas reservas energéticas mais rapidamente. $\mathrm{O}$ mesmo não foi observado em temperatura de $8^{\circ} \mathrm{C}$, quando os nematóides permanecem praticamente imóveis. A ação da glicerina pode ter influenciado na proteção contra a perda de reservas energéticas a $28^{\circ} \mathrm{C}$, quando os JIs infectantes se movimentam bastante, no entanto, a $8^{\circ} \mathrm{C}$ a mortalidade dos JIs pode estar mais associada a sua adaptação a baixas temperaturas do que ao gasto de energia.
O uso da glicerina pode potencializar a sobrevivência de JIs desses NEPs em condições de armazenamento a $28^{\circ} \mathrm{C}$, porém, de acordo com ANDALó et al. (2005), as porcentagens de sobrevivência e infectividade que foram obtidas a $16^{\circ} \mathrm{C}$ por até 180 dias foram maiores do que as encontradas nesse estudo na temperatura de $28^{\circ} \mathrm{C}$ com glicerina. Com isso, o uso da glicerina como agente conservante é útil em casos em que o nematóide ficará em temperaturas altas, ou caso não exista controle da temperatura.

A baixa disponibilidade de oxigênio pode reduzir a sobrevivência dos nematóides (GLAZER, 2002), e sua complementação para aumentar a sobrevivência deles em suspensão aquosa durante o armazenamento poderá ser empregada (ANDALóet al., 2006). Assim, além dessa condição para aumentar a sobrevivência desses nematóides, pode-se usar a temperatura e a adição de substância conservante.

A sensibilidade de algumas espécies à variação de temperatura, a suscetibilidade a contaminantes microbianos e a toxicidade de agentes antimicrobianos são fatores que influenciam na qualidade do armazenamento em água (GREWAL, 2000). Com isso, apesar da dificuldade de armazenamento ser um dos maiores entraves para a expansão do uso de NEPs como bioinseticidas, estudos que avaliem os fatores que afetam a sobrevivência desses organismos têm permitido que o armazenamento seja realizado de forma mais adequada. Assim, o uso de uma substância que prolongue a sobrevivência e mantenha a infectividade, para uma posterior liberação no campo em programas de controle de insetos, é desejável.

\section{REFERÊNCIAS}

ANDALÓ, V.; MOINO JUNIOR, A.; MOLINA ACEVEDO, J.P.; CAVALCANTI, R.S.; CARVALHO,

F.A. Efeito da temperatura e concentração na sobrevivência de nematóides entomopatogênicos em condições de armazena-mento, visando seu uso no controle microbiano de pragas. Boletín de Sanidad Vegetal Plagas, v.31, n.2, p.253-265, 2005.

\section{ANDALÓ, V.; CAVALCANTI, R.S.; MOLINA}

ACEVEDO, J.P.; MOINO JUNIOR, A.; MAGALHÃES,

F.H.L. Influência da aeração no armazenamento de nematóides entomoptogênicos (Rhabditida) em condições de laboratório. Nematologia Brasileira, v.30, n.1, p.45-50, 2006.

BROADBENT, A.B.; OLTHOF, T.H.A. Foliar application of Steinernema carpocapsae (Rhabditida:

Steinernematidae) to control Liriomyza trifolii (Diptera:

Agromyzidae) larvae in chrysanthemums.

Environmental Entomology, v.24, n.2, p.431-435, 1995. 
BROWN, I.M.; GAUGLER, R. Temperature and humidity influence emergence and survival of entomopathogenic nematodes. Nematologica, v.43, n.5, p.363-375, 1997.

DEMPSEY, C.M.; GRIFFIN, C.T. Phased activity in Heterorhabditis megidis infective juveniles. Parasitology, v.124, n.6, p.605-613, 2002.

DUTKY, S.R.; THOMPSON, J.V.; CANTWE, G.E. A technique for the mass propagation of the DD-136 nematode. Journal of Insect Pathology, v.6, n.4, p.417-422, 1964.

FITTERS, P.F.L.; GRIFFIN, C.T. Spontaneous and induced activity of Heterorhabditis megidis infective juveniles during storage. Nematology, v.6, n.6, p.911-917, 2004.

FLANDERS, K.L.; MILLER, J.M.; SHIELDS, E.J. In vivo production of Heterorhabditis bacteriophora 'Oswego' (Rhabditida: Heterorhabditidae), a potencial biological control agent for soil-inhabiting insects in temperate regions. Journal of Economic Entomology, v.89, n.2, p.373380, 1996.

GLAZER, I. Survival biology. In: GAUGLER, R. (Ed.). Entomopathogenic nematology. Wallingford: CABI Publishing, 2002. chap.8, p.169-188.

GREWAL, P.S. Anhydrobiotic potencial and long-term storage of entomopathogenic nematodes (Rhabditida: Steinernematidae). International Journal for Parasitology, v.30, n.14, p.995-1000, 2000.

HASS, B.; DOWNES, M.J.; GRIFFIN, C.T. Persistence of four Heterorhabditis spp. isolates in soil: role of lipid reserves. Journal of Nematology, v.34, n.2, p.151-158, 2002.

HATAB, M.A.; GAUGLER, R. Lipids of in vitro cultured Heterorhabditis bacteriophora. Biological Control, v.15, n.3, p.113-118, 1999.

KAYA, H.K.; STOCK, P. Techniques in insect nematology. In: LACEY, L. (Ed.). Manual of techniques in insect pathology. New York: Academic Press, 1997. chap.6, p.281-324.

LEWIS, E.E.; SELVAN, S.; CAMPBELL, J.F.; GAUGLER, $R$. Changes in foraging behaviour during the infective stages of entomopathogenic nematodes. Parasitology, v.110, n.5, p.583-590, 1995.
LEWIS, E.E.; SHAPIRO-ILAN, D. Host cadavers protect entomopathogenic nematodes during freezing. Journal of Invertebrate Pathology, v.81, n.1, p.25-32, 2002.

MENTI, H.; PATEL, M.N.; WRIGHT, D.J.; PERRY, R.N. Lipid utilisation during storage of the entomopathogenic nematodes Steinernema feltiae and Heterorhabditis megidis from Greece and the UK. Nematology, v.5, n.1, p.31-37, 2003.

MOLINA J.P.; LÓPEZ, N.J.C. Producción in vivo de tres entomonematodos con dos sistemas de infección en dos hospedantes. Revista Colombiana de Entomología, v.27, n.1-2, p.73-78, 2001.

PARRA, J.R.P. Criação de insetos para estudos com patógenos. In: Alves, S.B. (Ed.). Controle microbiano de insetos. Piracicaba: FEALQ, 1998. cap.35, p.10151037.

PATEL, M.N.; STOLINSKI, M.; WRIGHT, D.J. Neutral lipids and the assessment of infectivity in entomopathogenic nematodes: observations on four Steinernema species. Parasitology, v.114, n.6, p.489-496, 1997.

WESTERMAN, P.R. Aggregation of entomopathogenic nematodes, Heterorhabditis spp. and Steinernema spp., among host insects at 9 and $20^{\circ} \mathrm{C}$ and effects on efficacy. Journal of Invertebrate Pathology, v.73, n.2, p.206213, 1999.

WHITE, G.F. A method for obtaining infective nematode larvae from cultures. Science, v.66, p.302-303, 1927.

WRIGHT, D.J.; GREWAL, P.S.; STOLINSKI, M. Relative importance of neutral lipids and glycogen as energy stores in dauer larvae of two entomopathogenic nematodes, Steinernema carpocapsae and Steinernema feltiae. Comparative Biochemistry and Physiology. Part A, v.118, n.2, p.269273, 1997.

WRIGHT, D.J.; PERRY, R.N. Physiology and biochemistry . In: GAUGLER, R. (Ed.). Entomopathogenic nematology. Wallingford: CABI Publishing, 2002. chap.7, p.145-168.

Recebido em 5/3/07

Aceito em 20/8/08 